\title{
Is the recent increment in attributable deaths to type- 2 diabetes (T2D) associated with the latest chikungunya outbreak in a major epidemic area in Brazil?
}

\author{
Luciano Pamplona de Góes Cavalcanti ${ }^{11]}$, Sara Mendes D'angelo ${ }^{[1]}$, \\ Daniele Rocha Queiroz Lemos ${ }^{[2]}$, Francisca Kalline de Almeida Barreto ${ }^{[1],}$ \\ André Machado Siqueira ${ }^{[3]}$ and Fabio Miyajima ${ }^{[4],[5]}$
}

\author{
[1]. Programa de Pós-Graduação em Saúde Pública, Universidade Federal do Ceará, Fortaleza, CE, Brasil. \\ [2]. Centro Universitário Christus, Fortaleza, CE, Brasil. \\ [3]. Instituto Nacional de Infectologia Evandro Chagas, Fundação Oswaldo Cruz, Rio de Janeiro, RJ, Brasil. \\ [4]. Centro de Pesquisas e Desenvolvimento de Medicamentos, Universidade Federal do Ceará, Fortaleza, CE, Brasil. \\ [5]. Institute of Translational Medicine, University of Liverpool. Liverpool, United Kingdom.
}

\begin{abstract}
Introduction: Chikungunya virus (CHIKV) can negatively influence outcomes in patients with pre-existing conditions. We investigated the association between the recent CHIKV outbreak and increased type 2 diabetes (T2D)-attributable deaths. Methods: Monthly averages of T2D-attributable deaths between 2001 and 2016 were determined and compared to the equivalent data for 2017 and the recent CHIKV outbreak. Results: CHKV outbreak peaked in April 2017 with 4,394.4 cases/100,000 inhabitants, while T2D-attributable deaths in the same period increased by $35.2 \%$. Conclusions: T2D-attributable deaths significantly increased compared to the previous data, which overlapped with CHIKV incidence. The pathophysiology of this association warrants further investigations.
\end{abstract}

Keywords: Chikungunya. Diabetes. Arbovirus. Deaths.

Similar to most developing countries, the prevalence of type 2 diabetes (T2D) in Brazil has been continuously increasing, with the prevalence of $10.3 \%$ in $2012^{1}$. Patients with diabetes are at a greater risk to develop clinical complications following infections (e.g., tuberculosis and melioidosis), thus significantly adding to the existing burden in healthcare systems in low- and middle-income nations ${ }^{2}$. Several infections interact with T2D by compromising glucose metabolism, thus aggravating patient conditions and leading to increased mortality ${ }^{3}$. More than threequarters of patients with T2D live in developing countries ${ }^{3}$, where many infections are endemic. A recent review on T2D interaction with neglected infections, while presenting evidence of detrimental effect of dengue infection, failed to identify any literature on the consequences of Chikungunya (CHIKV) ${ }^{3}$.

CHKV was first described in 1952 in South Tanzania ${ }^{4}$. In Brazil, during the epidemic seasons in 2016 and 2017, more than 300,000 cases were reported, which in fact represent

Corresponding author: Dr. Luciano Pamplona de Góes Cavalcanti. e-mail: pamplona.luciano@gmail.com

Received 14 November 2017

Accepted 6 February 2018 a significant understatement since diagnostic tests are not universally available, particularly in remote regions. In the same period, 300 attributable deaths were confirmed, and while the risk of death has been estimated to be approximately 1:1,000 cases $^{5}$, the number of actual fatalities arising from CHKV is more likely higher since under-diagnosis of CHKV infections ${ }^{6}$ has directly impacted on the assessment of deaths by indirect causes $^{7-9}$. In the Federal State of Ceará, in the Northeast great region of Brazil, the first cases occurred in 2015 culminating with a large outbreak in 2017 where the incidence was more than $1,460.6$ cases per 100,000 inhabitants with 150 confirmed attributable deaths. The median death age of these subjects was 77 years, a population segment that inherently displays a higher prevalence of metabolic diseases like T2D.

Given that CHIKV can negatively influence metabolism in T2D patients and further derange glucose homeostasis, we sought to investigate, through an ecological approach, the association between a CHKV outbreak and a recent upsurge in the number of deaths attributable to T2D in the same area.

We determined the historical mean number of monthly deaths attributed to T2D (ICD10:E10-E15) between 2001 and 2016 in the State of Ceara. Next, we compared these numbers 
with: a) The respective monthly figures for 2017. b) The monthly incidence figures of CHKV in the state in 2017.

Between 2001 and 2016, the historical monthly mean of deT2D-attributable deaths was 154 and remained generally stable over the years with small seasonal variability. Conversely, during the CHKV outbreak season in 2017, the number of T2Dattributable deaths increased by $35.2 \%$ in April 2017 when compared with the historical data. This increase coincided with the peak of CHKV incidence, with a recorded 4,394.4 cases per 100,000 inhabitants in the same month. Considering only the first 6 months of the current year, CHIKV incidence significantly increased by $17.95 \%$ (173 deaths) as compared to the historical data (Figure 1).

These figures are likely to be an underestimate since the 2017 statistics are still being computed. Such a sudden spike in the number of deaths attributed to a non-communicable chronic disease led us to conjecture the manifestation of an acute trigging factor with the CHKV outbreak as an important element. Reports documenting the progression of T2D patients toward diabetic ketoacidosis, a life-threatening condition, have been found following CHKV infection ${ }^{10}$ in individuals who have had the need to scale-up anti-diabetic medication ${ }^{11}$ and others who had severe CHKV requiring hospitalization ${ }^{12,13}$. Our study highlights the need for thorough investigations of secondary causes, because of the fact that $\mathrm{CHKV}$ incidence is increasing worldwide and its consequences are still not fully clarified.
With the presence of biological plausibility, the pathophysiology of this association remains elusive, by either worsening the glucose metabolism or the diabetes could modify the course of infection. A probable hypothesis is that CHKV deregulates the balance of key cytokines, debilitating an already vulnerable immune system. For instance, the malfunctioning of type I interferon response in T2D patients could hamper response to $\mathrm{CHKV}$, thus leading to increased viral load and severity of the disease ${ }^{14}$. However, T2D-attributable deaths are difficult to associate with the CHKV incidence due to substantial inter-individual differences in CHKV disease progression and the inherent complexity of investigating an unpredictable disease that largely affects the elderly ${ }^{15}$. Another complication is related to $\mathrm{CHKV}$ infection leading to chronic musculoskeletal symptoms in approximately $50 \%$ of cases, which are frequently treated with either nonsteroidal anti-inflammatory drugs (NSAIDs) or glucocorticoids, whose continuous use has been associated with aggravation of T2D.

Comprehensive investigations on the interactions between CHKV infection and T2D progression are pivotal to establish a strategic agenda and promote actions to 1) elucidate the mechanistic basis of this association; 2) enhance management protocols for at-risk subjects; 3 ) evaluate visual and kidney complications arising from T2D aggravation; 4) stewardship on the rational use of corticosteroids and NSAIDs; and 5) assess the impact and economic costs of T2D in CHKV endemic zones.

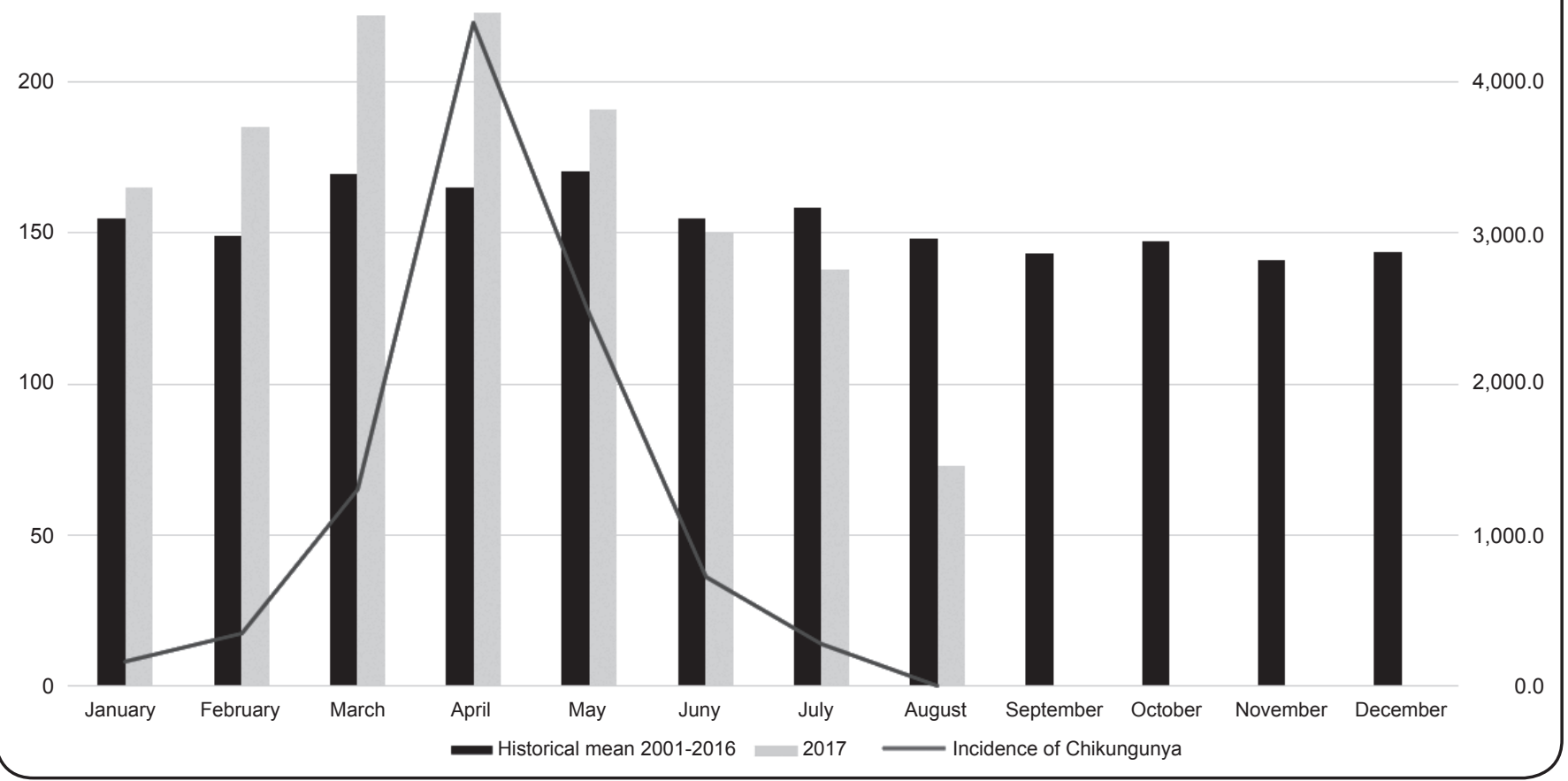

FIGURE 1: Historical mean of attributable deaths to T2D per calendar month (2001-2016) versus corresponding data for 2017 until August $^{*}$ and incidence of CHIKV virus infection in the same period in the State of Ceara. T2D: type-2 diabetes; CHIKV: Chikungunya virus *Data collection for T2D attributable deaths performed until mid-August 2017. 


\section{Conflict of interest}

The authors declare that there is no conflict of interest.

\section{REFERENCES}

1. Bertoldi AD, Kanavos P, França GV, Carraro A, Tejada CA, Hallal PC, Ferrario A, et al. Epidemiology, management, complications and costs associated with type 2 diabetes in Brazil: a comprehensive literature review. Global Health. 2013;9(62):1-12.

2. Korbel L, Spencer JD. Diabetes Mellitus and Infection: An Evaluation of Hospital Utilization and Management Costs in the United States. J Diabetes Complications. 2015;29(2):192-5.

3. Crevel RV, Vijver SV, Moore DAJ. The global diabetes epidemic: what does it mean for infectious diseases in tropical countries? Lancet Diabetes Endocrinol. 2017;5(6):457-68.

4. Wahid B, Ali A, Rafique S, Idrees M. Global expansion of chikungunya virus: mapping the 64-year history. Int J Infect Dis. 2017;58:69-76.

5. Caglioti C, Lalle E, Castilletti C, Carletti F, Capobianchi MR, Bordi L. Chikungunya virus infection: an overview. New Microbiol. 2013;36(3):211-27.

6. Freitas A, Zuben APB, Cavalcanti LPG, Donalísio MR. Excess Mortality Related to Chikungunya Epidemics in the Context of Cocirculation of Other Arboviruses in Brazil. Plos Currents. 2017;1:1-13.

7. Brito CAA. Alert: Severe cases and deaths associated with Chikungunya in Brazil. Rev Soc Bras Med Trop. 2017;50(5):585-9.
8. Mavalankar D, Shastri P, Bandyopadhyay T, Parmar J, Ramani KV. Increased mortality rate associated with chikungunya epidemic, Ahmedabad, India. Emerg Infect Dis. 2008;14(3):412-5.

9. Beesoon S, Funkhouser E, Kotea N, Spielman A, Robich RM. Chikungunya fever, Mauritius, 2006. Emerg Infect Dis. 2008;14(2):337-8.

10. Tolokh I, Laux T, Kim D. Case Report: A Case of Diabetic Ketoacidosis Following Chikungunya Virus Infection. Am J Trop Med Hyg. 2015;93(2):401-3.

11. Jean-baptiste E, Oettingen JV, Larco P, Raphaël F, Larco, NC, Cauvin MM, et al. Chikungunya Virus Infection and Diabetes Mellitus: A Double Negative Impact. Am J Trop Med Hyg, 2016;95(6):1345-50.

12. Crosby L, Perreau C, Madeux B, Cossic J, Armand C, HerrmannStorke C, et al. Severe manifestations of chikungunya virus in critically ill patients during the 2013-2014 Caribbean outbreak. Int J Infect Dis. 2016;48:78-80.

13. Sissoko D, Malvy D, Ezzedine K, Renault P, Moscetti F, Ledans $\mathrm{M}$, et al. Post-Epidemic Chikungunya Disease on Reunion Island: Course of Rheumatic Manifestations and Associated Factors over a 15-Month Period. PLoS Negl Trop Dis. 2009;3(3):1-6.

14. Summers KL, Marleau AM, Mahon JL, Mcmanus R, Hramiak I, Singh B. Reduced IFN-alpha secretion by blood dendritic cells in human diabetes. Clin Immunol, 2006;121(1):81-89.

15. Cavalcanti LPG, Freitas ARR, Brasil P, Cunha RV. Surveillance of deaths caused by arboviruses in Brazil: from dengue to chikungunya. Mem Inst Oswaldo Cruz. 2017;112(8):583-85. 